AS05

006

\title{
Symptomatic intracranial hemorrhage related to Estimated versus Measured Body Weight in intravenous thrombolysis
}

\section{BACKGROUND}

In acute ischemic stroke, under- or

overestimation of body weight can lead

to dosing errors of recombinant tissue

plasminogen activator (rt-PA) with

consequent reduced efficacy or

increased risk of hemorrhagic

complications. Measurement of body

weight (MBW) is more accurate than

estimation of body weight (EBW) but

potentially leads to longer door-to-

needle times (DNT). We assessed if

weight modality is associated with (I)

symptomatic intracranial hemorrhage

(sICH) (II) clinical outcome and (III) DNT.

\section{CONCLUSIONS}

Our study provides the largest multicenter cohort study to date assessing the association between weight modality (EBW or MBW) with $\mathrm{sICH}$, clinical outcome and DNT. We did not find evidence that weight modality (EBW or MBW) to determine rt-PA dose in IVT eligible patients is associated with these outcome parameters

\section{METHODS}

Consecutive patients treated with IVT between 2009-2016 from 14 hospitals (Figure 1) were included. Baseline characteristics and outcome parameters were retrieved from medical records. We defined sICH according to the ECASSIII definition and clinical outcome was assessed with the modified Rankin Scale (mRS). The association of weight modality and outcome parameters was estimated with regression analyses.

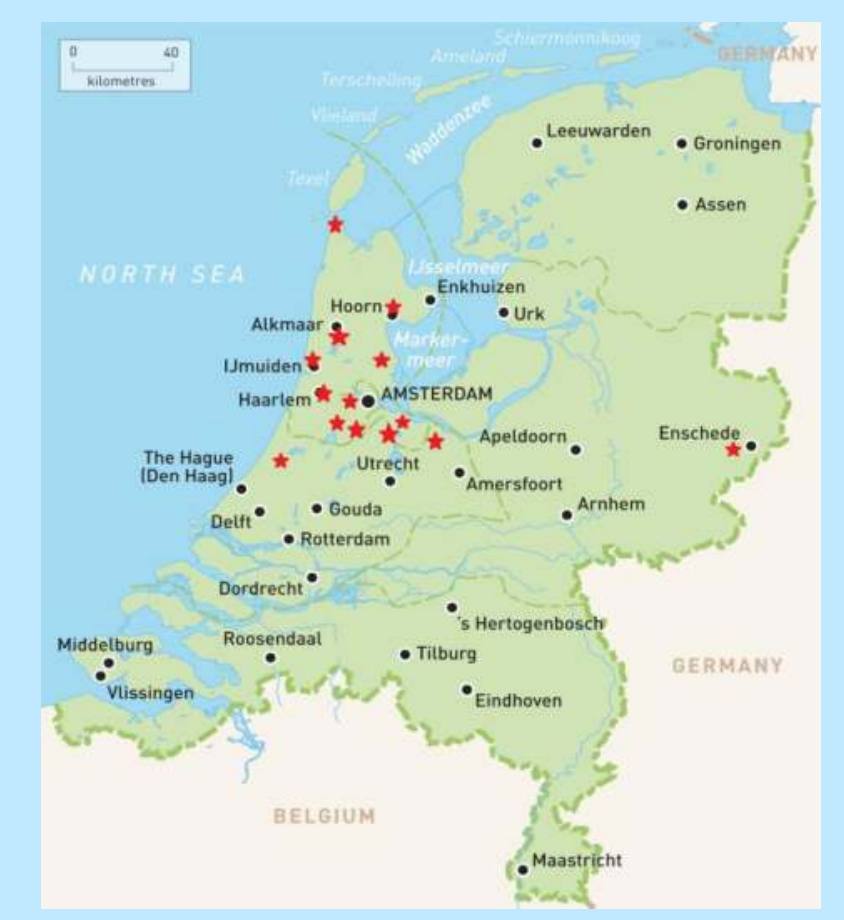

Figure 1.

\section{TABLES}

\begin{tabular}{|c|c|c|}
\hline Variables & $\begin{array}{l}\text { Measured body } \\
\text { weight }(n=2048)\end{array}$ & $\begin{array}{l}\text { Estimated body } \\
\text { weight }(n=2753)\end{array}$ \\
\hline \multicolumn{3}{|l|}{ Patient characteristics } \\
\hline Age at stroke, years - mean $( \pm \mathrm{SD})$ & $70( \pm 14)$ & $71( \pm 14)$ \\
\hline Male sex $-n(\%)$ & $1122(54.8)$ & $1502(54.6)$ \\
\hline \multicolumn{3}{|l|}{ Vascular risk factors } \\
\hline Atrial fibrillation $-n(\%)$ & $175(8.6)$ & 376 (14.1) \\
\hline Diabetes mellitus $-n(\%)$ & $309(15.3)$ & $469(17.5)$ \\
\hline Hypertension - $n(\%)$ & $840(41.5)$ & $1355(50.7)$ \\
\hline Hyperlipidemia - $n(\%)$ & $238(11.8)$ & $841(31.6)$ \\
\hline Coronary artery disease $-\mathrm{n}(\%)$ & $410(20.3)$ & $507(19.0)$ \\
\hline Peripheral vascular disease $-n(\%)$ & $112(5.6)$ & $156(5.8)$ \\
\hline Prior TIAstroke - $n(\%)$ & $528(26.2)$ & $660(24.7)$ \\
\hline \multicolumn{3}{|l|}{ Medication } \\
\hline Antiplatelets $-\mathrm{n}(\%)$ & $449(37.9)$ & $638(37.0)$ \\
\hline Anticoagulation $-n(\%)$ & $35(3.0)$ & $65(3.8)$ \\
\hline \multicolumn{3}{|l|}{ Admittance } \\
\hline Systolic BP, mmHg, mean $( \pm \mathrm{SD})$ & $156( \pm 25)$ & $156( \pm 26)$ \\
\hline Diastolic $B P, m m H g$, mean $( \pm S D)$ & $86( \pm 17)$ & $85( \pm 27)$ \\
\hline NIHSS, median [IQR] & $7[4-12]$ & $6[3-12]$ \\
\hline $\mathrm{ODT}, \min$ - median [IQR] & 69 [45-115] & 69 [45-112] \\
\hline \multicolumn{3}{|l|}{ IVT-volume (IVT/year) } \\
\hline High volume $(\geq 50)-n(\%)$ & $1121(54.7)$ & $2181(79.2)$ \\
\hline Medium volume (25-49) $-n(\%)$ & $656(32.0)$ & $470(17.1)$ \\
\hline Low volume (s24) - n (\%) & $271(13.2)$ & $102(3.7)$ \\
\hline$C T$ available in the $E R$ & $927(45.3)$ & $407(14.8)$ \\
\hline
\end{tabular}

Table 1. $\mathrm{TIA}=$ transient ischemic attack; $\mathrm{BP}=$ blood pressure; NIHSS = National Institute of Health Stroke Scale; ODT = onset-to-door time; IVT = intravenous thrombolysis; $\mathrm{CT}$ = computed tomography scan; $\mathrm{ER}=$ emergency room.

\section{RESULTS}

A total of 4801 (95\%) patients were included (Figure 2). Five hospitals used MBW ( $n=1753)$, six hospitals used EBW $(n=2325)$ and three hospitals changed from EBW to MBW during the study period.

In 2048 of the patients (43\%) MBW was used and in 2753 (57\%) EBW.

EBW-patients were slightly older and they had more cardiovascular risk factors (atrial fibrillation, diabetes mellitus, hypertension and

hyperlipidemia) (Table 1). Other known predictors for sICH (sex, NIHSS score, blood pressure and onset-to-door time) did not differ between the EBW and the MBW group.

Estimation of body weight was not associated with increased sICH risk (adjusted OR = 1.16; 95\% Cl 0.84-1.62), favourable outcome (adjusted OR = $0.95 ; 95 \% \mathrm{Cl} 0.73-1.23)$ or with DNT (adjusted B $=0.32 ; 95 \% \mathrm{Cl}-1.62-2.25$ ) (Table 2).

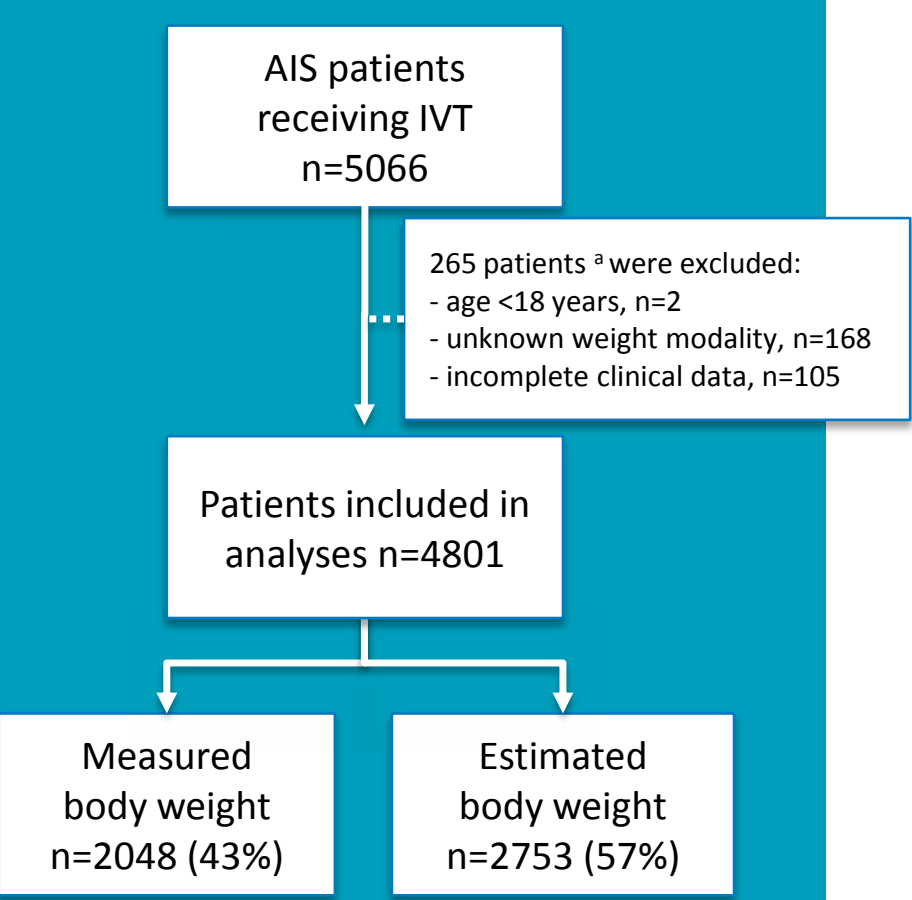

Figure 2. Study flowchart. a Ten patients had incomplete data and an unknown weight modality. AIS = acute ischemic stroke; IVT = intravenous thrombolysis 